\title{
Measurement uncertainty in calibration and compliancy testing of PCR and qPCR thermal cyclers
}

\author{
Mary $\operatorname{Span}^{1, *}$, Marc Verblakt ${ }^{2}$, and Tom Hendrikx ${ }^{1}$ \\ ${ }^{1}$ CYCLERtest BV, Rötscherweg 61, 6374 XW Landgraaf, the Netherlands \\ ${ }^{2}$ CelsiusLabs, Rötscherweg 61, 6374 XW Landgraaf, the Netherlands
}

\begin{abstract}
Both testing and medical laboratories use thermal cycler and real-time thermal cycler equipment for Polymerase Chain Reaction (PCR) based DNA testing. These thermal cyclers require regular calibration to ensure proper functioning. Currently, a variety of thermal cycler calibration and verification methods exits. Most of these methods mimic the PCR reaction, are not traceable to the International System of Units (SI) and connected to substantial measurement uncertainties, impeding compliancy reporting. Therefore these methods are inapt for application in metrology. This paper discusses a universal temperature calibration method for thermal cyclers that can be applied to any brand and model PCR and $\mathrm{qPCR}^{\#}$ thermal cycler, irrespective of the heating and cooling technology used. The calibration method and low associated measurement uncertainties do allow meaningful compliancy reporting to meet the requirements of ISO/IEC 17025, ISO 15189, ILAC P10 and ILAC G8. In addition, the paper offers effective practical solutions to determine PCR method based specifications, rather than thermal cycler manufacturer specifications, to link the PCR method to the thermal cycler, in order to qualify if the instrument is suitable for the intended use.
\end{abstract}

\section{Introduction}

With the increasing number of testing and medical testing laboratories obtaining an ISO/IEC 17025 or ISO 15189 accreditation the need for metrological traceable calibration methods increases. For general laboratory equipment, like pipettes and balances, established and standardized calibration methods exist since several decades $[1,2]$. For more specific and less abundantly used equipment, like thermal cyclers, no standardized calibration methods are currently available. Therefore a wide variety of thermal cycler calibration and verification methods is currently used. The majority of these thermal cycler calibration and verification methods have been developed from a molecular biologist rather than from a metrologist point of view. As a result, most of these methods mimic the PCR reaction, are not traceable to the International System of Units (SI) and connected to substantial measurement uncertainties, impeding compliancy reporting. Therefore these methods are inapt as metrological traceable calibration methods.

To offer a solution to ISO/IEC 17025 and ISO 15189 accredited labs a universal metrological traceable PCR and qPCR thermal cycler calibration method was developed based on an initial literature study and additional research. The universal thermal cycler method was ultimately validated via an interlaboratory comparison.

\section{Evaluation of existing methods}

An initial literature study on existing thermal cycler calibration and verification methods was done to evaluate existing methods and to identify potential candidates or elements for a universal metrological traceable thermal cycler calibration method. The two parameters which were taken into account in the evaluation were (1) the traceability to the International System of Units (SI) and (2) the measurement uncertainty, as these two aspects are crucial for their use in metrology.

Traceability to SI can only be obtained via an unbroken chain of comparisons going back to an international standard, ending at a primary standard for the realisation of SI units $[3,4]$. The traceability shall be up to the level of the thermal cycler and not merely up to the level of the thermal cycler calibration system.

An adequately low measurement uncertainty can only be obtained by excluding or minimizing as many systematic and random measurement errors as possible. In metrology it is commonly accepted that test uncertainty ratio (TUR) shall be at least a factor 3 to 4 less than the maximal acceptable tolerance (e.g. manufacturer or method based specifications) [5]. In sensitive PCR reactions as little as $0.5{ }^{\circ} \mathrm{C}$ deviation in temperature can already result in different, incorrect or failing PCR results [6]. Therefore, the expanded combined measurement uncertainty of thermal cycler

Corresponding author: marys@cyclertest.com

(C) The Authors, published by EDP Sciences. This is an open access article distributed under the terms of the Creative Commons Attribution 
calibration systems shall be $\leq 0.15{ }^{\circ} \mathrm{C}(k=2)$ to enable meaningful compliancy reporting.

The existing thermal cycler calibration and verification methods can be divided into biochemical and physical methods. The evaluation of the methods was done per method type as similar methods share characteristics.

\subsection{Biochemical methods}

The biochemistry or chemistry based methods, like thermographic liquid crystals based optical temperature verification discs [7], PCR based temperature verification kits $[8,9,10]$, temperature sensitive PCR methods $[6,11,12]$ and melt curve based qPCR methods [13], heavily reply on the use of biochemicals or chemicals. Biochemicals and chemicals can seldomly be obtained as $100 \%$ pure substance. As a result, a large part of the measurement uncertainty of the method is pre-determined by the purity of the (bio)chemicals used, rendering (bio)chemical methods inapt as thermal cycler compliancy testing method, simply due to their large measurement uncertainties. In addition, (bio)chemical methods are typically lacking traceable to SI, rendering the (bio)chemical methods as well inapt as metrological traceable calibration method. No potential candidates or elements for a universal metrological traceable thermal cycler calibration method were identified among the biochemical methods.

\subsection{Physical methods}

Physical methods rely on measuring directly physical quantities. Physical methods can be divided in contact and non-contact methods.

Non contact methods, like for example infrared thermography, are connected to substantial measurement uncertainties and therefore excluded from the evaluation. Contact methods, based on temperature sensors, and can be divided in two subcategories; (1) in-tube methods and (2) in-well methods.

Temperature sensor based methods can be made traceable to SI and are therefore a good candidate for metrological traceable thermal cycler calibration methods. The measurement uncertainty ultimately determines the extent to which these methods are suitable for compliancy testing. Therefore the measurement uncertainties of the different methods were assessed in detail to identify potential sources of systematic and random errors as most publications lack detailed information on the measurement uncertainty of the method.

\subsubsection{In-tube methods}

The concept of in-tube methods is mimicking the PCR reaction and measuring the temperature inside the tube like it occurs during the PCR. These in-tube methods use temperature sensors in water or oil filled PCR tubes, which are positioned in the wells of the thermal cycler $[6,11,14,15,16]$

The evaluation showed that the published in-tube methods contained flaws, causing both systematic and random measurement errors, each contributing to the measurement uncertainty. For each of the flaws the magnitude of the systematic and/or random error was estimated.

The first flaw is caused by filling the tubes with water instead of PCR reaction mix and not correcting the water volume for the difference in the heat capacity between water and PCR reaction mix. A PCR mix typically consist of water, buffering components, salts, dNTPs (deoxynucleotide triphosphates), template DNA, oligonucleotides, DNA polymerase, glycerol and in some cases DMSO (dimethylsulfoxide) and a fluorescent intercalating due. Especially the glycerol and DMSO strongly affect the heat capacity of the water. The heat capacity of a PCR mix is $5-10 \%$ lower than the heat capacity of water (3.90-3.95 vs. $4.18 \mathrm{~J} / \mathrm{g} * \mathrm{~K})$, leading to a systematic negative measurement error of about $0.05{ }^{\circ} \mathrm{C}$.

The second flaw is caused by the sensor-in-tube assembly. A large part of the sensor wires are exposed to air inside and outside the tube and can therefore extract heat from the sensor and lose it to the environment. This stem effect results in a systematic negative error of about $0.05-0.10^{\circ} \mathrm{C}$, depending on the diameter of the wires and the environmental temperature.

The third flaw error is caused by the calibration of the temperature sensors. The temperature sensors are calibrated as bare temperature sensors without the sensor-in-tube assembly. This leads to an underestimation of the thermal mass of the complete sensor-in-tube assembly leading to a systematic negative error of $0.1-0.5{ }^{\circ} \mathrm{C}$. The underestimation of the thermal mass also affects the response time (Tau) of the sensorin-tube assembly, which leads to an underestimation of the measurement uncertainty in the dynamic part of a measurement, when measuring for example ramp rates and overshoots.

The fourth flaw is caused by positioning the tubes in the wells without using a contact medium like oil or thermal paste. Depending on the fit of the tube to the well this contact error is $0.1-0.5^{\circ} \mathrm{C}$ [17].

The fifth flaw is caused by the tubes. $0.1 \mathrm{ml}$ or 0.2 $\mathrm{ml}$ PCR tubes are available from a large number of different manufacturers, each having their own manufacturing specifications, tube dimensions, tube angles and wall thicknesses. Therefore tubes of different brands have different tube-to-well fits and different heat transfer capacities. Dependent on the reproducibility of production and the number of cavities of the injection mould not all tubes from one bag are identical copies of each other and therefore a substantial tube to tube variability can be present. This random error can vary substantially and is estimated to vary between 0.3 and $1.0{ }^{\circ} \mathrm{C}$.

The sixth flaw is caused by evaporation of water from the tube. Depending on the model and make of the tube, evaporation rates range between 5 and $15 \%$, during a 40 cycle PCR temperature protocol (40 cycles of $30 \mathrm{~s}$ $95{ }^{\circ} \mathrm{C}, 30 \mathrm{~s} 60{ }^{\circ} \mathrm{C}, 30 \mathrm{~s} 72{ }^{\circ} \mathrm{C}$ ) [17]. When using similar 
protocols for the calibration of a thermal cycler, the volume of water will decrease during cycling temperature protocol, leading to a decrease in thermal mass during the calibration and therefore a decrease in response time of the sensor during the thermal cycler calibration. This error is a gradually increasing positive error over time and reaches its maximum value at the end of the calibration. The magnitude of the error is about $0.05{ }^{\circ} \mathrm{C}$. If the same temperature is measured in consecutive cycles this error may also be interpreted as non reproducibility of the thermal cycler from cycle to cycle. However, block based thermal cyclers have turned out to be very stabile from cycle to cycle, if pre-heated before use [17].

The seventh flaw is caused by leakage of liquid via capillary force along the sensor wires. The effect is most profound when using oil as liquid medium [17]. Due to this capillary leakage the mass of the sensor construction will decrease over time, which will lead to overestimation of the temperature and a positive systematic error. The loss of mass, when using oil, happens gradually but steady. So the thermal cycler calibration system will show a drift of about $0.05{ }^{\circ} \mathrm{C}$ between annual recalibrations, which is not caused by drift of the sensor, but loss of mass of the sensor-in-tube assembly.

The magnitude of the different systematic and random errors, each contributing to the combined expanded measurement uncertainty of the thermal cycler calibration can differ between the different methods described. In total they add up to expanded combined measurement uncertainties of over $2{ }^{\circ} \mathrm{C}(k=2)$, rendering in-tube methods inapt as thermal cycler compliancy testing method, simply due to their large measurement uncertainties.

\subsubsection{In-well methods}

The concept of in-well methods is performing measurements in a maximum standardized way with a low measurement uncertainty. These in-well methods use temperature sensors which are positioned directly in the wells of the thermal cycler, using in some cases a contact medium like oil or thermal paste $[18,19,20,21$, $22,23,24]$. The reported expanded combined measurement uncertainties of the calibration systems used in these in-well methods vary between 0.10 and $0.30{ }^{\circ} \mathrm{C}(k=2)$ are herewith substantially lower than the assessed measurement uncertainties of the in-tube methods. Combined with the potential to make these methods traceable to SI, in-well methods are more suitable candidates for compliancy reporting.

The evaluation showed that some published in-well methods contained flaws, causing both systematic and random errors, contributing to the measurement uncertainty.

The first flaw is caused by the mass of the sensor assembly. The mass of a semi-skirted 96-well plate filled with $25 \mathrm{ul} \mathrm{PCR}$ mix is between 25 and $30 \mathrm{~g}$. This mass corresponds with a normal load during a PCR run. Some systems have masses up to $90 \mathrm{~g}$ [18], which is 3 times more. This high mass results in long stabilisation times, impeding dynamic measurements. The measurement protocol often involves several cycles and data is only recorded during the last cycle of the protocol when equilibrium has been reached. The method is therefore not representative of the PCR process and will lead to negative systematic errors at early time points in the measurement. Also the height of overshoots will be underestimated.

The second flaw is caused by the number of sensors used. Methods using less than 12 sensors [19, 21] underestimate the uniformity of a thermal cycler due to insufficient coverage of the block and therefore results in a negative systematic error when measuring uniformity. The magnitude of this error depends on the non uniformity of the block and can vary between $0.1^{\circ} \mathrm{C}$ and $0.5{ }^{\circ} \mathrm{C}[25]$.

The third flaw is caused by the sensor locations in the block, eventually combined with the number of sensors. Methods that exclude corner locations [20], edge locations $[18,21]$ or the middle of the heating elements of a thermal cycler underestimate the uniformity of a thermal cycler as these locations often belong to the coldest and hottest locations in a thermal cycler. This results in a negative systematic error when measuring uniformity. The magnitude of this error depends on the non uniformity of the block and can vary between $0.1{ }^{\circ} \mathrm{C}$ and $0.5^{\circ} \mathrm{C}$ [25].

The forth flaw is caused by measuring with the heated lid open. Methods that measure with the heated lid open [20] typically report lower average block temperatures and higher non uniformities. This effect is mainly caused by the block loosing heat to the environment, rather than then the heated lid being part of the heating mechanism of the block. The effect is most profound around the edges of the block and can vary between $0.2{ }^{\circ} \mathrm{C}$ and $0.4{ }^{\circ} \mathrm{C}$ and is dependent on the environmental temperature [25]. Although open lid measurements are not incorrect, they are less representative of the PCR process than closed lid measurements. Not each thermal cycler can be measured with the heated lid closed. So it is recommended to mention in the calibration certificate if the measurement has been performed with opened or closed heated lid.

Based on these findings above it was concluded that a good potential candidate for a universal metrological traceable thermal cycler calibration method is a physical in-well method with a expanded combined measurement uncertainty of $\leq 0.15^{\circ} \mathrm{C}(k=2)$, measuring in at least $12.5 \%$ of the wells (12 wells in a 96-well block), covering corner, edge and central locations and measuring with the heated lid closed and active.

\section{Additional points of consideration}

In order to ultimately develop a universal metrological traceable thermal cycler calibration method that can be applied to any brand and model thermal cycler and that is capable of measuring accuracy, uniformity, ramp rates, hold times and overshoots correctly, additional research was done. The optimal number of sensors 
combined with the ideal sensor layout was assessed via a risk based approach, a universal temperature protocol was formulated and the sampling frequency was examined.

\subsection{Number of sensors and sensor layout}

To determine the optimal number of sensors and ideal sensor layout a risk-based approach was taken. The aim was to minimize the risk of missing a hot or cold spot. A thermal cycler block is heated and cooled by one or more thermo-electric elements, so-called Peltier elements, that each heat and cool part of a thermal cycler block. The hottest temperatures may be expected in the middle of the Peltier elements and the coldest spots on the transition lines between Peltier elements, around the edges of the block and at the corners of the block. Around the edges and at the corners the block a well can lose heat in respectively one or two directions. Central wells are surrounded by 8 other wells and therefore the risk of losing heat to the environment is lower.

Market information showed that the number of Peltier elements can vary between 1 and 24 in blockbased thermal cyclers and is typically not known by the user of a thermal cycler. Therefore the thermal cycler shall be considered as a black box and the sensor layout shall offer a solution to each number of Peltier elements that can be expected. Based on this information and the assessment of where to expect hot and cold spots a number of criteria have been set for the sensor layout in a 96-well block.

The criteria for the sensor layout were:

a) sensors shall be located evenly distributed over the block in at least $12.5 \%$ of the wells (12 wells in a 96-well block) and not include directly adjacent well locations

b) sensors locations shall include 4 corner locations

c) sensor locations shall include 4 edge locations (1 along each edge of the block),

d) sensor locations shall include Peltier transitions in case of 2, 4 or 8 Peltier elements

e) sensor locations shall include the middle of the 96well block

f) sensor locations shall include the middle of the Peltier element in case of 2 or 4 Peltier elements

g) each Peltier element shall be covered by at least 2 sensors in case of $\leq 8$ Peltier elements

When these criteria were combined with the different existing Peltier layouts each of the wells in a 96-well block could be categorized as low or high risk zone for hot or cold spots as shown in Figure 1 for thermal cyclers heated by $2,4,6$, or 8 Peltier elements. When the sensors were placed on the high risk zones it turned out that a 16 sensor layout was the most optimal layout that is capable of measuring 2, 4, 6, 8 and 16 Peltier layouts with 1 or more sensors per Peltier element combined with corner, edge and central locations as shown in Figure 2a. The minimum layout that is capable of measuring 2 and 4 Peltier layouts was a 12 sensor layout as shown in Figure $2 b$.
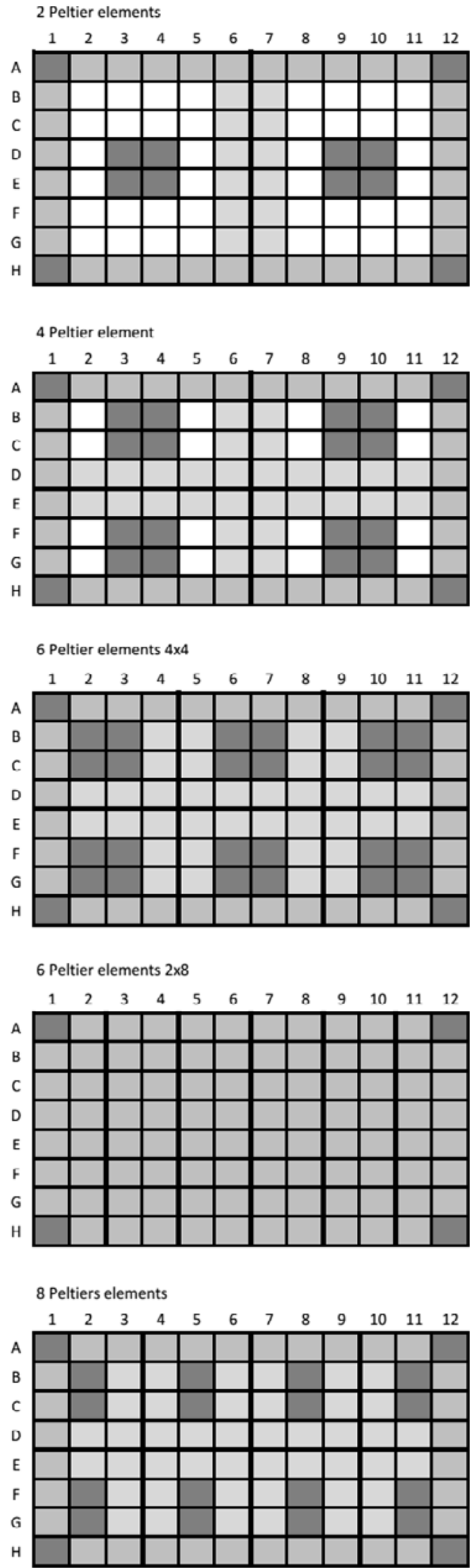

Fig. 1. High risk (dark grey), medium risk (medium grey) and low risk (light grey) wells in 96-well thermal cycler blocks heated by either $2,4,6$ or 8 Peltier elements. 
a.

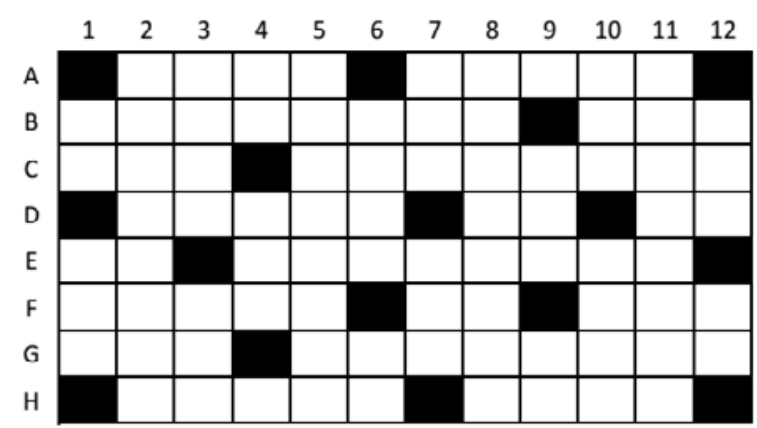

b.

96 well block 12 sensor minimal layout

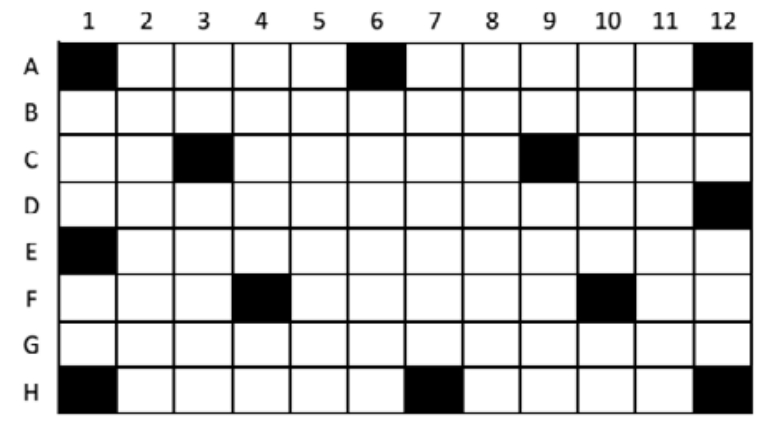

Fig. 2. Optimal (a) and minimal (b) sensor layouts for 96-well thermal cycler blocks.

The risk-based assessment has then also been applied to other thermal cycler block and heating chamber formats and has resulted in final sensor layout criteria as described in 5.1.

\subsection{Temperature protocol}

In order to be representative of the PCR reaction, the thermal cycler calibration temperature shall include temperatures close to the denaturation (typically between 94 and $98{ }^{\circ} \mathrm{C}$ ), annealing (typically between 50 and 65 ${ }^{\circ} \mathrm{C}$ ) and elongation (typically $72{ }^{\circ} \mathrm{C}$ ) temperatures. Choosing exactly the PCR temperatures used shall be avoided, as this will result in a multitude of calibrations on the same thermal cycler for all different PCR assays used, resulting in high calibration costs. By choosing for example standard temperatures like $30^{\circ} \mathrm{C}$ or $40^{\circ} \mathrm{C}, 50$ ${ }^{\circ} \mathrm{C}, 60{ }^{\circ} \mathrm{C}, 70{ }^{\circ} \mathrm{C}, 90{ }^{\circ} \mathrm{C}$ and $95{ }^{\circ} \mathrm{C}$ the complete PCR reaction range, including eventual reverse transcriptase steps, is covered. This protocol can be applied to any thermal cycler and also allows comparing the temperature performance of different thermal cyclers. The denaturation temperature shall be measured in heating mode, the annealing temperature in cooling mode and the elongation temperature in heating mode. Each of the temperature steps shall be held for a certain time span to allow the thermal cycler to reach equilibrium and to measure if the thermal cycler is able to hold the temperature for a longer time. Most thermal cyclers have reached thermal equilibrium after 30s [17].
During the development of the method it was noticed that thermal cycler calibration results were more reproducible if the thermal cycler was pre-heated directly before the calibration with a $60 \mathrm{~s} 30{ }^{\circ} \mathrm{C}, 60 \mathrm{~s} 95$ ${ }^{\circ} \mathrm{C}, 60$ s $30{ }^{\circ} \mathrm{C}$ pre-heat.

The ultimately formulated optimal protocol was the following: $60 \mathrm{~s} 30^{\circ} \mathrm{C}, 60 \mathrm{~s}, 95^{\circ} \mathrm{C}, 30 \mathrm{~s} 30^{\circ} \mathrm{C}$ (pre-heat) 60 s $30{ }^{\circ} \mathrm{C}, 60 \mathrm{~s} 95^{\circ} \mathrm{C}, 60 \mathrm{~s} 30^{\circ} \mathrm{C}, 60 \mathrm{~s} 90^{\circ} \mathrm{C}, 60 \mathrm{~s} 50$ ${ }^{\circ} \mathrm{C}, 60 \mathrm{~s} 70{ }^{\circ} \mathrm{C}, 60 \mathrm{~s} 50{ }^{\circ} \mathrm{C}$ (calibration).

Air-cooled thermal cyclers may not be able to get down to $30{ }^{\circ} \mathrm{C}$ at elevated room temperatures. In that case $40{ }^{\circ} \mathrm{C}$ or $45{ }^{\circ} \mathrm{C}$ may be used instead of $30{ }^{\circ} \mathrm{C}$. Changing this minimum temperature will, however, impact the measured maximum heat and cool rate as the temperature difference between minimum and maximum temperature will be smaller.

\subsection{Sampling frequency}

In order to be capable of correctly measuring heat rates, cool rates, overshoots and undershoots the measurement frequency of the thermal cycler calibration system shall be sufficiently high. Low measurement frequencies result in missing the top of the overshoot or undershoot and incorrect determination of the maximum heat rate or cool rate. Thermal cyclers from the early days heat with heat rates of $1{ }^{\circ} \mathrm{C} / \mathrm{s}$. Most modern block-based thermal cyclers use heat rates between $2{ }^{\circ} \mathrm{C} / \mathrm{s}$ and $5{ }^{\circ} \mathrm{C} / \mathrm{s}$. Figure 3 shows the difference between a measurement frequency of $2 \mathrm{~Hz}$ and $4 \mathrm{~Hz}$ when measuring the overshoot of a thermal cycler with a heat rate of $5{ }^{\circ} \mathrm{C} / \mathrm{s}$. So in order to measure heat rates and overshoots correctly a measurement frequency of at least $4 \mathrm{~Hz}$ is required.

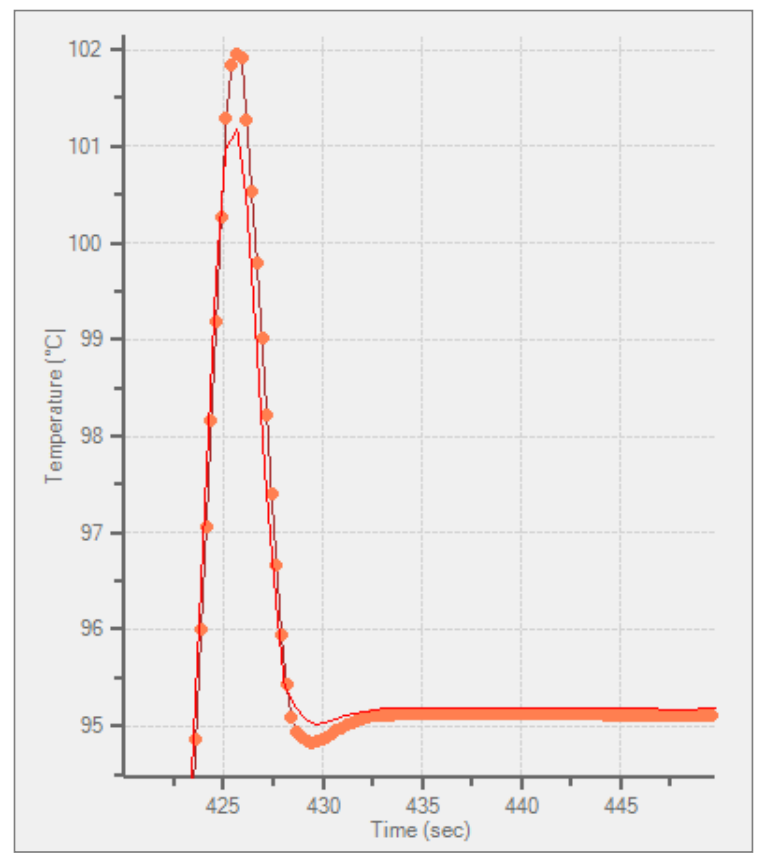

Fig. 3. Overshoot at $95{ }^{\circ} \mathrm{C}$ measured with a sampling frequency of $2 \mathrm{~Hz}$ (straight line) $4 \mathrm{~Hz}$ (dotted line) 


\section{Universal calibration method}

Based on the literature study and additional research a universal metrological traceable thermal cycler calibration method that can be applied to any brand and model PCR and qPCR thermal cycler and that is capable of measuring accuracy, uniformity, ramp rates, hold times and overshoots correctly was formulated. Criteria were set for the thermal cycler calibration system, the sensor locations, the data acquisition and the environmental conditions.

\subsection{Temperature calibration system}

The thermal cycler temperature calibration system shall meet the following criteria:

a) multi-sensor system with adequate number of temperature sensors to measure simultaneously;

- in at least $12.5 \%$ of the wells for heating blocks and chambers $\leq 96$ wells

- in at least 12 wells for heating blocks and chambers $>96$ wells

b) capable of recording the heated lid temperature with at least 1 temperature sensor (when thermal cycler uses a heated lid and can be measured closed);

c) capable of recording with multiple temperature sensors simultaneously in order to allow measuring the temperature distribution across the block (also called uniformity);

d) capable of recording temperatures dynamically with a sampling frequency of at least $4 \mathrm{~Hz}$ in order to allow capturing fast ramp rates;

e) capable of recording the temperature over the complete temperature range of the PCR reaction;

f) capable of being calibrated, traceable to SI, over at least the complete temperature range of the PCR reaction;

g) read-out resolution of at least $0.01{ }^{\circ} \mathrm{C}$;

h) expanded combined measurement uncertainty of temperature calibration system of $\leq 0.15^{\circ} \mathrm{C}(k=2)$.

\subsection{Temperature sensor locations}

The thermal cycler temperature sensor locations shall meet the following criteria:

For any format thermal cycler:

a) The temperature sensors shall be located evenly distributed over the heating block or chamber in at least $12.5 \%$ of the wells for heating blocks or chambers $\leq 96$ wells or 12 wells for heating blocks and chambers $>96$ wells

For rectangular heating block based thermal cyclers:

a) The temperature sensors shall be located evenly distributed over the heating block or chamber in at least $12.5 \%$ of the wells for heating blocks or chambers $\leq 96$ wells or 12 wells for heating blocks and chambers > 96 wells;

b) The locations shall include 4 corner locations for heating blocks $>2$ columns or rows of wells or 2 corner locations for heating blocks $\leq 2$ columns or rows of wells; c) The locations shall include at least 1 edge location for each edge of the heating block for heating blocks $\geq 6$ wells;

d) The locations shall include at least 2 central locations for heating blocks $>18$ wells and at least 4 central locations for heating blocks $>48$ wells.

Examples of sensor layouts for 24, 48, 96 and 384 well thermal cycler blocks meeting these criteria are shown in Figure 4.
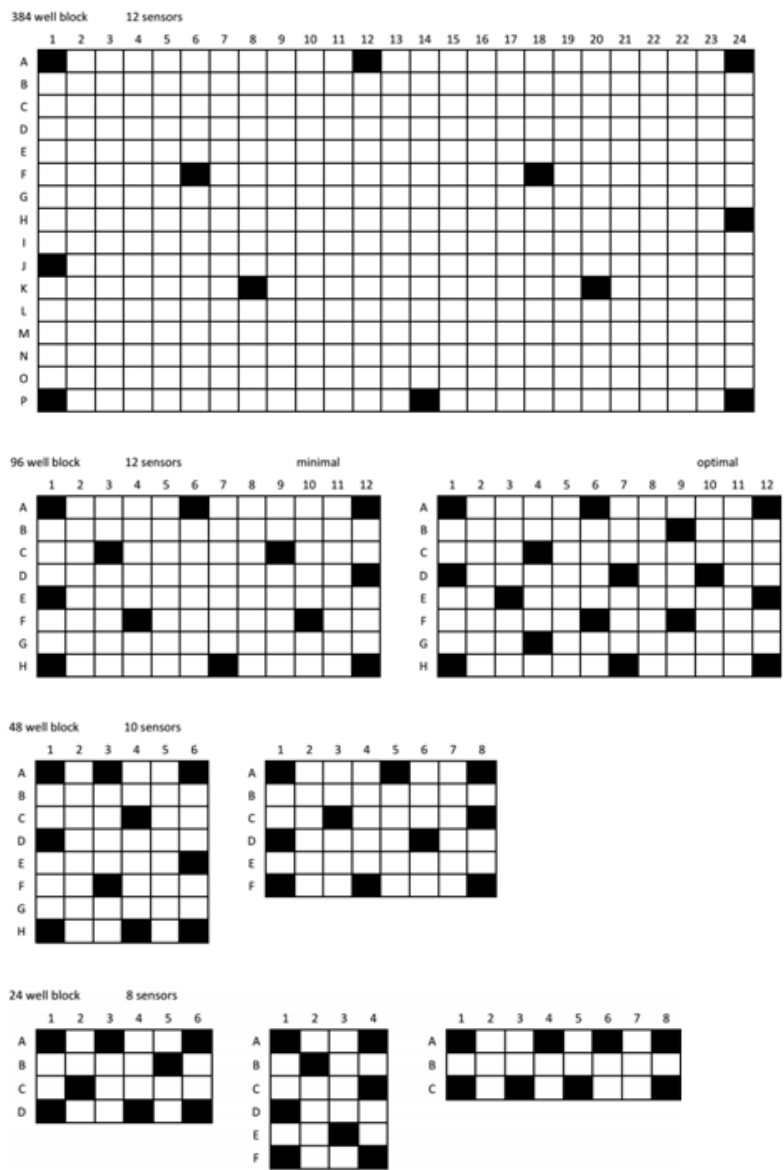

Fig. 4. Example sensor layouts for 384, 96, 48 and 24 well thermal cycler blocks.

\subsection{Data acquisition}

The data acquisition of the thermal cycler temperatures shall meet the following criteria:

a) The sampling frequency shall be at least $4 \mathrm{~Hz}$;

b) The temperature protocol shall cover the complete temperature range of the PCR reaction protocol and include at least a minimum, middle and maximum temperature and ideally universal temperatures close to the denaturation, annealing and elongation temperatures;

c) The temperatures close to the denaturation shall be measured in heating mode, temperatures close to annealing shall be measured in cooling mode and temperatures close to elongation shall be measured in heating mode in order to measure representative 
of the PCR process and in order to determine overshoots and undershoot in the correct direction;

d) The temperature protocol shall include a heating ramp from minimum to maximum temperature and a cooling ramp from maximum to minimum temperature in order to determine the maximum heat and cool rate;

e) The temperature protocol shall include a pre-heat including the minimum and maximum temperature to pre-heat the heat-sink of the thermal cycler;

f) The hold times shall be representative for the PCR process in which temperatures quickly alternate, but be at minimum 30 s to allow stabilisation of the thermal cycler;

g) In case of block based thermal cyclers, the heated lid shall be set at a higher temperature than the block at any moment in time during the temperature protocol.

An example of a temperature protocol meeting these criteria is: $60 \mathrm{~s} 30{ }^{\circ} \mathrm{C}, 60 \mathrm{~s}, 95^{\circ} \mathrm{C}, 30 \mathrm{~s} 30{ }^{\circ} \mathrm{C}$ (pre-heat) 60 s $30{ }^{\circ} \mathrm{C}, 60 \mathrm{~s} 95{ }^{\circ} \mathrm{C}, 60 \mathrm{~s} 30^{\circ} \mathrm{C}, 60 \mathrm{~s} 90{ }^{\circ} \mathrm{C}, 60 \mathrm{~s} 50$ ${ }^{\circ} \mathrm{C}, 60 \mathrm{~s} 70{ }^{\circ} \mathrm{C}, 60 \mathrm{~s} 50{ }^{\circ} \mathrm{C}$ (calibration).

\subsection{Environmental conditions}

The environmental conditions shall meet the following criteria to not invalidate the thermal cycler calibration results:

a) The environmental temperature shall be between 14 and $28^{\circ} \mathrm{C}$;

b) The relative humidity shall be between 10 and $90 \%$;

c) The thermal cycler shall not be placed in any airflow (draught or air conditioning flow) or close to heat generating instruments.

\section{Validation of universal method}

\subsection{Uncertainty calculation}

For the validation of the universal method a 16 sensor, thermistor based, thermal cycler calibration system was constructed that was used for the systematic assessment of the factors influencing the result, through variation of controlled parameters and via evaluation of the measurement uncertainty of the results in order to calculate and estimate type A and B errors of the universal thermal cycler calibration method. The expanded combined measurement uncertainty of the thermal cycler calibration system was calculated and appeared to be as low as $0.09^{\circ} \mathrm{C}(k=2)$.

\subsection{Interlaboratory comparison}

The validation was completed by participation to an interlaboratory comparison [25]. In this interlaboratory comparison both in-well and in-tube methods, methods measuring with opened and closed heated lid and methods using between 8 and 16 sensors were included. The reported results included average block temperature and uniformity at $30{ }^{\circ} \mathrm{C}, 50{ }^{\circ} \mathrm{C}, 60{ }^{\circ} \mathrm{C}, 70{ }^{\circ} \mathrm{C}, 90^{\circ} \mathrm{C}$ and $95{ }^{\circ} \mathrm{C}, 30 \mathrm{~s}$ after the target temperature of the thermal cycler block was reached. The calculated $|\mathrm{En}|$ values were between 0.03 and 0.17 for average block temperature and between 0.11 and 0.83 for uniformity.

The |En| values proof that the formulated universal method is measuring without systematic errors and that the calculated expanded combined measurement uncertainty is correctly determined

\section{Method based compliancy testing}

Ultimately, the calibration results are compared to specifications to evaluate if the PCR or qPCR thermal cycler is suitable for the intended use. Under ISO/IEC 17025 and ISO 15189 accreditation the measurement uncertainty shall be taken into account when comparing to specifications, as described in ILAC G8 [26]. When the measurement uncertainty is taken into account the resulting acceptance zone is smaller than the specification zone (Figure 5). Very narrow specification zones can therefore lead to no acceptance zones as the uncertainty zone overlaps the complete specification zone. To allow meaningful compliancy reporting it is therefore crucial to set specifications based on the method as the final goal is to evaluate if the thermal cycler is suitable for the method used and not if the thermal cycler meets manufacturer specifications. In practise method based specifications can be much wider than manufacturer specifications. For robust PCR methods the accuracy specification can be up to $+/-1{ }^{\circ} \mathrm{C}$ and the uniformity specification can be up to $<1.5{ }^{\circ} \mathrm{C}$, whereas most modern thermal cyclers are specified at $+/$ $0.25^{\circ} \mathrm{C}$ for accuracy and $<0.5^{\circ} \mathrm{C}$ for uniformity.

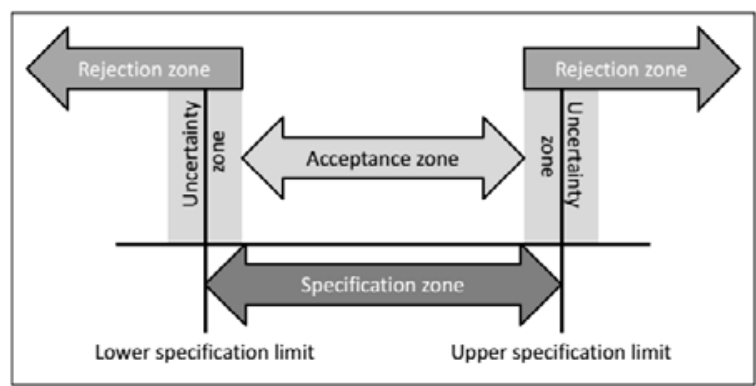

Fig. 5. Acceptance zone versus specification zone.

The simplest way to determine method based specifications is to first run a gradient PCR at denaturation temperature while keeping the annealing and elongation temperature constant. Then run a gradient PCR at annealing temperature, while keeping the denaturation and elongation temperature constant. The elongation is less critical as it functions over a very wide temperature range and therefore does not need to be tested. The gradient PCRs will allow determining the minimum and maximum denaturation and annealing temperatures that still lead to a correct PCR result. These maximum and minimum temperatures represent the method specifications. 
The hot and cold spots and resulting uniformity of a thermal cycler can be determined via the universal thermal cycler calibration method and then be compared to the method specifications, taking the expanded combined measurement uncertainty into account. This can be done for each thermal cycler to be used for a particular method. This way of compliancy testing allows qualifying each thermal cycler as suitable for a particular method and makes PCR validation runs per thermal cycler superfluous. This way of compliancy testing does in addition not require thermal cyclers to be identical copies, but allows some variation between thermal cyclers, as long as the total uniformity plus the measurement uncertainty does not exceed the method specification limits.

\section{Conclusions}

The formulated universal thermal cycler calibration method can be applied to any brand and model PCR and qPCR thermal cycler and is capable of measuring accuracy, uniformity, ramp rates, hold times and overshoots correctly. The universal thermal cycler calibration method is metrological traceable and the thermal cycler calibration system has an expanded combined measurement uncertainty $\leq 0.15{ }^{\circ} \mathrm{C} \quad(k=2)$. Therefore this method is suitable as metrological traceable calibration method which allows meaningful compliancy testing and meets the requirements of the ISO/IEC 17025, ISO 15189, ILAC P10 and ILAC G8. When compliancy testing will be done versus method based specifications it will also allow qualifying a thermal cycler as suitable for the method used.

\section{References}

1. ISO 8655-6:2002, Piston operated volumetric apparatus - Part 6: Gravimetric methods for the determination of measurement error.

2. EN 45501:2015, Metrological aspects of nonautomatic weighing instruments

3. JCGM 200:2012, International Vocabulary of Metrology - Basic and General Concepts and Associated Terms VIM, $3^{\text {rd }}$ edition, issued by BIPM, IEC, IFCC, ILAC, ISO, IUPAC, IUPAPA and OIML

http://www.bipm.org/en/publications/guides/vim.ht $\mathrm{ml}$

4. ILAC-P10:01/2013, ILAC policy on the Traceability of Measurement Results http://ilac.org/publicationsand-resources/ilac-policy-series/

5. K. Bennett, H. Zion, Metrology concepts: Understanding Test Uncertainty Ratio (TUR) (2005) http://www.transcat.com/media/pdf/TUR.pdf

6. I. Yang, Y.-H. Kim, J.-Y. Byun, S.-R. Park, Analytical Biochemistry, 338, 192-200 (2005)

7. Rotor-Disc OTV Handbook https://www.qiagen.com/us/resources/resourcedetail ?id=5bb3981b-cb8d-4480-99ffb70ebf9b0115\&lang=en

8. Taqman RNase $\mathrm{P}$ instrument verification plate https://tools.thermofisher.com/content/sfs/manuals/4 314333.pdf

9. Congen SureCycle Manual 08/2002E

10. CyclerCheck Instructions for use http://www.baghealthcare.com/fileadmin/user_upload/PDF_Diagno stik/IFUs/HISTO_TYPE_Happy/IFUCYCLERCHECK-V9-2016-EN.pdf

11. Schoder, 2005, J Clin Microbiology, 2724-2728 in tube method PCR method

12. G.C. Saunders, J. Dukes, H.C. Parkes, J.H. Cornett, Clin chem, 47 :1, 47-55 (2001)

13. Von Keyserling, H., Bergmann, M. Wiesel, A. Kaufmann, BioTechniques, 51, 179-184 (2011)

14. Y.-H. Kim, I. Yang, Y-S. Bae, S.-R. Park, BioTechniques, 44, 495-505 (2008)

15. D. Schoder, A. Schmalwieser, G. Schauberger, K. Kuhn, J. Hoorfar, M. Wagner, Clin Chem, 49:6, 960-963 (2003)

16. Thermal cycler calibration service http://www.totalq.gr/en/services/calibrations/temper ature-relative-humidity.html

17. Internal report GENO-tronics In-tube versus in-well calibrations

18. On-site Temperature Verification Service https://www.thermofisher.com/nl/en/home/productsand-services/services/instrument-qualificationservices/compliance-and-validation/on-sitetemperature-verification.html

19. On-site thermal cycler temperature calibration service http://www.lbtblt.be/nl/etalonage/validation.php

20. On-site thermal cycler temperature calibration service http://www.idoslab.com/servicios.html

21. PCR cycler service https://nl.vwr.com/store/content/externalContentPag e.jsp;jsessionid=ps2jvBDF2b7j38L6n2wmqmL6.est ore5b?path=/nl.vwr.com/nl_NL/pcr_cycler_service.j sp

22. Thermal cycler testing service http://www.isq.pt/EN/services/metrology/\#tab-id-10

23. DRIFTCON Operations Manual http://www.cyclertest.com/Downloads.aspx

24. MTAS Operations Manual http://www.cyclertest.com/Downloads.aspx

25. Internal report CYCLERtest ILC Thermocycler calibration

26. ILAC-G8:03/2009, Guidelines on the Reporting of Compliance with Specification http://ilac.org/publications-and-resources/ilacguidance-series/ 